\title{
All-optical Inverter based on Polarization Switching in Long-wavelength VCSELs
}

\author{
A. Quirce ${ }^{1,2}$, J. R. Cuesta ${ }^{1}$, A. Hurtado ${ }^{3}$, K. Schires ${ }^{3}$, A. Valle ${ }^{1}$, L. Pesquera ${ }^{1}$, I. D. Henning, ${ }^{3}$ M. J. Adams ${ }^{3}$ \\ 1. Instituto de Física de Cantabria (CSIC-Universidad de Cantabria), Avda. Los Castros s/n, E39005, Santander, Spain. \\ 2. Departamento de Física Moderna, Universidad de Cantabria, Avda. Los Castros s/n, E39005, Santander, Spain.. \\ 3. School of Computer Science and Electronic Engineering, University of Essex, Wivenhoe Park, CO4 3SQ Colchester, U.K.
}

All-optical processing of high-speed signals is expected to be a key technology in future photonic networks. VCSELs are very promising devices for these applications due to their inherent advantages. Nonlinear transfer functions can be obtained by using optical injection because it strongly affects the transverse mode and polarization characteristics of VCSELs [1]. Optical injection can be used to obtain polarization switching (PS) of single transverse mode VCSELs. When the polarization of an injected optical field is orthogonal to that of the solitary VCSEL (orthogonal optical injection), PS and bistability are observed [2]. PS by optical injection is expected to be fast and thus suitable for application in all-optical signal-processing systems. In this work we report, for the first time to our knowledge, all-optical inversion operation using PS in a 1550nm single-mode VCSEL induced by orthogonal optical injection. We demonstrate all-optical inversion for NRZ signals at a bit rate of $2.5 \mathrm{~Gb} / \mathrm{s}$. An all-fiber system has been developed in order to inject the light from a tunable laser into a commercial $1550 \mathrm{~nm}-\mathrm{VCSEL}$ (RayCan, Co.). The free-running VCSEL emits in the fundamental transverse mode with a linear polarization ("parallel" polarization) over the whole current range. The subsidiary orthogonal polarization is shifted $0.48 \mathrm{~nm}$ to the long wavelength side with respect to the dominant one. Pulsed optical injection was obtained by using a pulse pattern generator and a Mach-Zhender intensity modulator.

Fig. 1 shows the results for an input signal of $2.5 \mathrm{~Gb} / \mathrm{s}$ non-return-to-zero (NRZ) data with a bit pattern "10111000011100011110110110100111". The wavelength detuning between the externally injected signal and the orthogonal polarization mode of the VCSEL is $0.05 \mathrm{~nm}$. The bias current applied to the VCSEL is $4 \mathrm{~mA}$ $\left(2.1 \mathrm{I}_{\mathrm{th}}\right)$. Fig. 1(a) shows the input signal with a rectangular waveform that arrives at the VCSEL. Figs. 1(b) and 1(c) show respectively orthogonal and parallel polarizations at the output of the device. The dynamical evolution of the parallel polarized power is shown in Fig. 1(c). Good waveform inversion is demonstrated with a $2.5 \mathrm{~Gb} / \mathrm{s}$ NRZ input signal when comparing Figs. 1(a) and (c). In contrast the power of the orthogonal polarization, shown in Fig. 1(b), shows unwanted peaks appearing just after the "10" bit transitions. These results suggest that use of the orthogonal polarization to obtain all-optical gating would suffer pattern-dependent error-rate degradation in a digital system: this requires further investigation. In conclusion, we report a first experimental demonstration of all-optical inversion at $2.5 \mathrm{~Gb} / \mathrm{s}$ with a $1550 \mathrm{~nm}$-VCSEL. We speculate that the speed of operation is ultimately limited by the relaxation oscillation frequency (ROF) of the device and hence we expect to obtain higher speeds $(10 \mathrm{~Gb} / \mathrm{s}$ or more) using devices with higher ROF values as VCSEL technology matures.

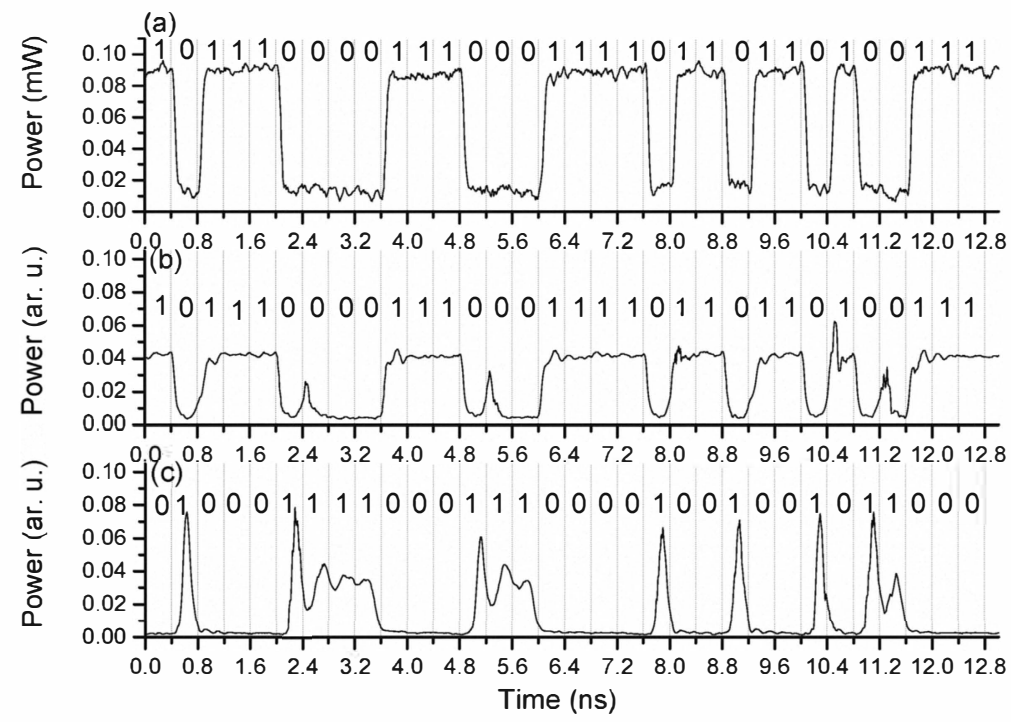

Fig. 1 Waveform inversion of a $2.5 \mathrm{~Gb} / \mathrm{s} \mathrm{NRZ} \mathrm{input} \mathrm{signal.} \mathrm{(a)} \mathrm{Input} \mathrm{light.} \mathrm{(b)} \mathrm{Power} \mathrm{of} \mathrm{the} \mathrm{orthogonal} \mathrm{polarization.} \mathrm{(c)}$ Power of the parallel polarization. The VCSEL is biased at $4 \mathrm{~mA}\left(2.1 \times \mathrm{I}_{\mathrm{th}}\right)$.

\section{References}

[1] F. Koyama, "Recent advances of VCSEL Photonics," J. Lightwave Technol. 24, 4502 (2006)

[2] A. Hurtado, A. Quirce, A. Valle, L. Pesquera, and M. J. Adams, "Power and wavelength polarization bistability with very wide hysteresis cycles in a 1550nm-VCSEL subject to orthogonal optical injection," Opt. Exp. 17, 23637 (2009). 